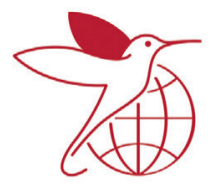

International

Diabetes

Federation

\title{
Effects of fructose-induced metabolic syndrome on rat skeletal cells and tissue, and their responses to metformin treatment
}

\author{
Juan Ignacio Felice ${ }^{a}$, León Schurman ${ }^{a}$, Antonio Desmond McCarthy ${ }^{a}$, Claudia Sedlinsky $^{a}$, \\ José Ignacio Aguirre ${ }^{b}$, Ana María Cortizo ${ }^{a, *}$ \\ ${ }^{a}$ Laboratorio de Investigaciones en Osteopatías y Metabolismo Mineral (LIOMM), Facultad de Ciencias Exactas, \\ Universidad Nacional de La Plata, Argentina \\ ${ }^{\mathrm{b}}$ Department of Physiological Sciences, University of Florida, Gainesuille, FL, USA
}

\section{A R T I C L E I N F O}

\section{Article history:}

Received 30 August 2016

Received in revised form

6 December 2016

Accepted 7 February 2017

Available online 14 February 2017

\section{Keywords:}

Metabolic syndrome

Fructose

Bone architecture

Osteoblasts

Metformin

\begin{abstract}
A B S T R A C T
Aims: Deleterious effects of metabolic syndrome (MS) on bone are still controversial. In this study we evaluated the effects of a fructose-induced MS, and/or an oral treatment with metformin on the osteogenic potential of bone marrow mesenchymal stromal cells (MSC), as well as on bone formation and architecture.

Methods: 32 male 8 week-old Wistar rats were assigned to four groups: control (C), control plus oral metformin (CM), rats receiving 10\% fructose in drinking water (FRD), and FRD plus metformin (FRDM). Samples were collected to measure blood parameters, and to perform pQCT analysis and static and dynamic histomorphometry. MSC were isolated to determine their osteogenic potential.

Results: Metformin improved blood parameters in FRDM rats. PQCT and static and dynamic histomorphometry showed no significant differences in trabecular and cortical bone parameters among groups. FRD reduced TRAP expression and osteocyte density in trabecular bone and metformin only normalized osteocyte density. FRD decreased the osteogenic potential of MSC and metformin administration could revert some of these parameters. Conclusions: FRD-induced MS shows reduction in MSC osteogenic potential, in osteocyte density and in TRAP activity. Oral metformin treatment was able to prevent trabecular osteocyte loss and the reduction in extracellular mineralization induced by FRD-induced MS.
\end{abstract}

(c) 2017 Elsevier B.V. All rights reserved.

\section{Introduction}

Metabolic syndrome (MS) is defined as the group of risk factors that predispose individuals to the development of type 2 Diabetes and cardiovascular disease [1]. This syndrome was first described by Reaven in 1988 and initially named "X
Syndrome" [2]. Since then, MS has been subjected to several revisions with the intention of providing a more suitable definition worldwide. Following this objective, in 2009 a harmonized definition of MS was published [3]. According to this joint statement, a diagnosis of MS is made when at least 3 of the 5 following risk factors are present: central obesity,

\footnotetext{
* Corresponding author at: LIOMM-Facultad de Ciencias Exactas, Universidad Nacional de La Plata, 47 y 115, 1900 La Plata, Argentina. E-mail address: cortizo@biol.unlp.edu.ar (A.M. Cortizo).
} 
elevated triglycerides, decreased HDL-cholesterol, elevated blood pressure, and elevated fasting glucose, with the inclusion of patients taking medication to manage hypertriglyceridemia, low HDL-cholesterol, hypertension and/or hyperglycemia [3].

The negative effect of Diabetes mellitus (DM) on the skeleton and its association with bone fractures is well established [4]. Patients with type $2 \mathrm{DM}$ and high bone mineral density, also show an increased rate of osteoporotic bone fractures [5]. However, less is known about the effects of MS on bone metabolism. Different reports reveal conflicting results regarding MS or its individual components, with bone mineral density and/or fracture risk $[6,7]$.

Western diets are rich in carbohydrates (e.g. fructose and sucrose) and saturated fats and are associated with MS and cardiovascular disease. Many MS animal models are based in the administration of high-carbohydrate or high-fat diets, to develop one or more of the characteristics of this syndrome [8]. In particular, high-fructose diets have been successfully used in animal models to develop MS, displaying classical clinical and metabolic changes that include: hypertension, glucose intolerance, hypertriglyceridemia, insulin resistance and obesity [9-11]. Rats fed a high-fructose diet show a systemic accumulation of advanced glycation end-products (AGEs) secondarily to the presence of hyperglycemia [12]. Our group and others have shown that AGEs trigger oxidative stress and inflammatory reactions, both of which are related to metabolic disorders as well as to impaired bone cell development and survival [13,14]. More recently, we have shown that fructose-induced MS rats display alterations in metaphyseal bone microarchitecture and defective bone fracture healing, possibly as a result of a deviation in the adipogenic/ osteogenic commitment of MSC due to an imbalance in the Runx2/PPAR $\gamma$ ratio [9].

Metformin is one of the most widely used agents for treatment of insulin resistance associated with type $2 \mathrm{DM}$ and MS. We have shown that metformin also enhances osteoblast proliferation, differentiation and mineralization in the UMR 106 and MC3T3E1 osteoblastic cell lines [15], and induces bone formation in vivo and ex vivo in normal rats and in partiallyinsulin-deficient diabetic rats [16].

Based on these previous observations, we hypothesize that fructose-induced MS alters osteoblast differentiation and thus, maintenance of normal bone architecture and that these effects can be prevented by metformin treatment.

\section{Materials and methods}

\subsection{Animals and experimental design}

A schematic representation of the experimental design is shown in Fig. 1. Thirty-two 8 week-old male Wistar rats (200-220 g) were housed in a temperature-controlled room at $23 \pm 3{ }^{\circ} \mathrm{C}$, with average humidity of $30-70 \%$, a $12: 12 \mathrm{~h}$ light: dark cycle and continuous access to standard rat laboratory chow (Asociación de Cooperativas Argentinas, Buenos Aires, Argentina) and beverage ad libitum. The experiments were carried out in conformity with the Guidelines on Handling and Training of Laboratory Animals published by the Universities Federation for Animal Welfare [17]. All experiments including animals were approved by the Institutional Ethics Committee ( $\mathrm{N}^{\circ}$ 001-05-15).

Animals were randomly divided into two groups of 16 animals. One of the groups received sterile water ad libitum. The other group was given a $10 \% \mathrm{w} / \mathrm{v}$ fructose solution (Biopack, Buenos Aires, Argentina) ad libitum until the end of the study $[9,18,19]$. After 14 days of treatment, half the rats from each group also received $100 \mathrm{mg} / \mathrm{kg} /$ day metformin (Química Montpellier, Buenos Aires, Argentina) added to their drinking water for the last 3 weeks of the study. Thus, four experimental groups of eight animals per group were set: control (C); control plus metformin (CM); fructose rich diet (FRD); and fructose rich diet plus metformin (FRDM). Treatment times were chosen based on previous reports showing that fructose administration to rats for at least 2 weeks induces metabolic changes that resemble human MS [10,18,20-25], as well as bone tissue alterations [9]. The metformin dose of $100 \mathrm{mg} /$ $\mathrm{kg} /$ day that we employed in the in vivo experiments was within the human therapeutic range as modified by the Guidance of FDA-CDER [26]. In addition, Choi and colleagues [27] have shown that plasma half-life for oral metformin in rats is $2 \mathrm{~min}$ (versus $5 \mathrm{~h}$ in humans), underscoring the need for significantly higher oral dosing in rats. We have also demonstrated that oral administration of metformin for 2 weeks induces significant changes in in vivo and in vitro osteogenic parameters $[15,16,28]$. Total treatment time was not prolonged any further to avoid insulin secretion impairment, as explained in Section 4.

Half of the rats from each group received subcutaneous fluorochromes injections to perform dynamic histomorphometry [29]. Briefly, ten days prior to sacrifice rats received

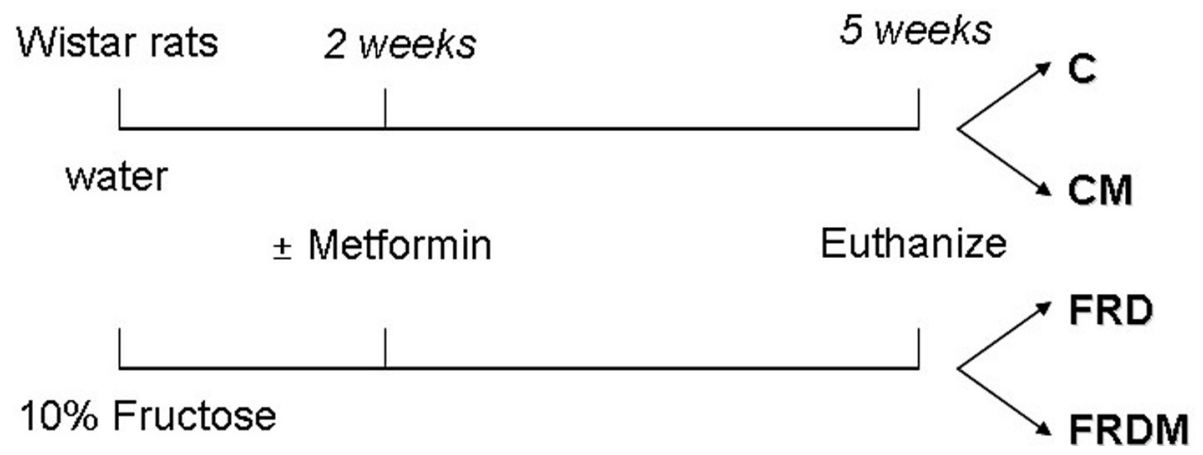

Fig. 1 - Schematic representation of the experimental design. C: control group; CM: control plus metformin treatment group; FRD: group of rats receiving fructose rich-diet and FRDM: group of rats receiving fructose rich-diet plus metformin treatment. 
demeclocycline hydrochloride $(15 \mathrm{mg} / \mathrm{kg}$ body weight; Sigma Chemical Co., St. Louis, MO), and three days before sacrifice they received calcein $(15 \mathrm{mg} / \mathrm{kg}$ body weight; Sigma Chemical Co., St. Louis, MO).

After 5 weeks of total treatment, rats were weighed and anaesthetized with ketamine hydrochloride/xylazine. Under anesthesia, blood samples were obtained from the inferior vena cava after which animals were euthanized by cervical dislocation.

Left and right femurs, left tibiae, and first and second lumbar vertebrae were dissected, measured using a digital caliper (Fischer Scientific), and processed as described in each section.

\subsection{Blood measurements}

Serum was separated from other blood components by centrifugation (3500 rpm, $15 \mathrm{~min}$ ). Glucose, triglycerides, cholesterol, insulin, and fructosamine concentrations were determined. Glucose, triglycerides and cholesterol were measured by commercial kits (Wiener Laboratories, Rosario, Argentina) with an automated clinical chemistry analyzer (Metrolab 2300 plus, Buenos Aires, Argentina). Insulin was determined by a rat-specific ELISA kit (ALPCO, New Hampshire, USA) and fructosamine was measured by a colorimetric kit (Biosystems, Barcelona, Spain). Insulin resistance was estimated with the Homeostasis Model Assessment-Insulin Resistance (HOMA-IR) index, using the formula ([Glucose $(\mathrm{mg} / \mathrm{dL})] \times[\operatorname{Insulin}(\mathrm{mU} / \mathrm{L})] / 2430)[30]$.

\section{3. $\quad \mathrm{QQCT}$ analysis of bone structural parameters}

Left femurs and tibiae, and second lumbar vertebrae from half of the rats per group were stripped of musculature and placed in $70 \%$ ethanol at $4^{\circ} \mathrm{C}$ until analysis. Bones were scanned using a Stratec XCT Research M instrument (Norland Medical Systems, Fort Atkinson, WI) equipped with software version 6.20. Two scans were performed in the long bones: the first one at the distal femoral or proximal tibial metaphyses ( $20 \%$ of total bone length), to assess parameters of trabecular bone, and the second one at the midshaft (diaphysis) ( $50 \%$ of total bone length) to evaluate parameters of cortical bone [29]. The first scanning sites were determined using the scout scan and were at the level of the secondary spongiosa. Analyzed structural variables were: total bone mineral content (totBMC), total bone mineral density (totBMD), trabecular bone mineral content (trBMC), and trabecular bone mineral density (trBMD) in the metaphysis, and cortical bone mineral content (cBMC), cortical bone mineral density (cBMD), cortical thickness, periosteal circumference, and endocortical circumference in the midshafts.

\subsection{Structural and static histomorphometric analyses of distal femoral metaphysis}

Distal metaphyses were processed as described in the previous section. Four $\mu \mathrm{m}$ thick sections were obtained with a Leica RM 2025 rotatory microtome (Leica Microsystems, Wetzlar, Germany). Sections were stained with von Kossa stain and counterstained with Tetrachrome (Sigma-Aldrich, St. Louis, MO) [31].

To avoid the primary spongiosa and endocortical surfaces, a region of interest was delimited between 0.5 and $4 \mathrm{~mm}$ from the growth plate, and $0.25 \mathrm{~mm}$ from the endocortical surfaces.

The following parameters were evaluated: trabecular bone volume (BV/TV, as a percentage of total volume), osteoid volume (OV/BV, as a percentage of trabecular bone volume), trabecular number (Tb.N, \#/mm), trabecular thickness (Tb. $\mathrm{Th}, \mathrm{mm}$ ), trabecular separation (Tb.S, $\mathrm{mm}$ ), osteoblastcovered surface (Ob.S/BS, as a percentage of trabecular bone surface), osteoclast-covered surface (Oc.S/BS, as a percentage of trabecular bone surface), and eroded surface (ES/BS, as the percentage of trabecular bone surface eroded by osteoclasts). All these parameters were measured with the Osteomeasure System (Osteometrics Inc., Atlanta, GA). The terminology used was based on recommendations by the Histomorphometry Nomenclature Committee of the American Society of Bone and Mineral Research [32].

\subsection{Dynamic histomorphometric analysis of distal femoral metaphysis}

Right femurs of fluorochrome injected rats were stripped of musculature and fixed in Neutral Buffered Formalin (NBF) for $24 \mathrm{~h}$. Distal metaphyses were dehydrated in ethanol solutions, embedded undecalcified in modified methyl methacrylate and sectioned longitudinally with a Leica RM 2025 rotatory microtome (Leica Microsystems, Wetzlar, Germany) to obtain $8 \mu \mathrm{m}$ thick samples as previously described [29]. Fluorochrome based data were collected using a Nikon Labophot-2 microscope (Nikon Instruments Inc., Melville, NY) and the Osteomeasure System (Osteometrics Inc., Atlanta, GA). The evaluated dynamic histomorphometric parameters included: mineralizing surface (MS/BS, calculated as the sum of the percentage of trabecular bone surface with double fluorochrome labels plus half of the percentage of trabecular bone surfaces with single fluorochrome labels), mineral apposition rate (MAR), and bone formation rate (BFR/BS, calculated by multiplying MS/BS by MAR). Longitudinal bone growth was calculated as the quotient of the distance between the second fluorochrome label in the primary spongiosa and the limit between hypertrophic and proliferative chondrocytes in the growth plate, and the time elapsed between the administration of the second label and sacrifice.

\subsection{Structural morphometric analysis in proximal femoral metaphysis}

After sacrifice, the right femora of non-fluorochrome-injected rats were used for histomorphometric analysis. The bones were fixed in Neutral Buffered Formalin (NBF) for $72 \mathrm{~h}$ and decalcified by consecutive immersions in 10\% EDTA (Biopack, Buenos Aires, Argentina), $\mathrm{pH}=7.0$. After decalcification, samples were dehydrated, embedded in paraffin and sectioned at five $\mu \mathrm{m}$ using an SM 2000R Leica microtome (Leica Microsystems, Wetzlar, Germany). Sections were stained with H\&E or processed for Tartrate Resistant Acid Phosphatase (TRAP) detection (Sigma, St. Louis, MO, USA). 
The H\&E sections were used to determine relative trabecular bone area, defined as the ratio between trabecular bone area and total tissue area Tb.Ar/T.Ar [\%]; and osteocyte density defined as the number of osteocytes per square millimeter of trabecular bone. Photographs were taken with a Micrometrics 519CU camera system on an Eclipse E400 Nikon microscope (Nikon, Tokyo, Japan). Images were analyzed using the ImageJ Program (http://www.macbiophotonics.ca/ imagej).

\subsection{Mesenchymal stromal cells (MSCs) isolation and incubation}

The MSCs were obtained from left femora of 4 rats per group. Immediately after dissection femoral epiphyses were sectioned and bone marrow cells were collected by flushing with Dulbecco's modified essential medium (DMEM) (Invitrogen, Buenos Aires, Argentina), through the medullary cavity under sterile conditions [33]. Resulting cell suspensions were seeded in $25 \mathrm{~cm}^{2}$ culture flasks and incubated in DMEM supplemented with 10\% fetal bovine serum (FBS) (Natocor, Córdoba, Argentina), and antibiotics (100 UI/mL penicillin, $100 \mu \mathrm{g} / \mathrm{mL}$ streptomycin) at $37{ }^{\circ} \mathrm{C}$ in a humidified atmosphere with $5 \%$ $\mathrm{CO}_{2}$ and $95 \%$ air. After $24 \mathrm{~h}$, culture medium was changed to remove non-adherent cells [28]. After this step, medium was changed twice a week. When cells reached confluence (1015 days), the cell monolayer was detached using 0.025\% trypsin (GIBCO, Invitrogen, Buenos Aires, Argentina) prepared in $1 \mathrm{~mm}$ EDTA and sub-cultured in tissue plates.

\subsection{Osteogenic differentiation of MSC}

MSC were seeded in 24-well plates at a density of $5 \times 10^{4}$ cells/ well and incubated with 10 FBS-DMEM supplemented with antibiotics at $37^{\circ} \mathrm{C}$ in a humidified atmosphere with $5 \mathrm{CO}_{2}$. After cells reached confluence, they were induced to differentiate into osteoblasts using an osteogenic medium (10 FBSDMEM containing $25 \mu \mathrm{g} / \mathrm{mL}$ ascorbic acid and $5 \mathrm{mmol} / \mathrm{L}$ sodium $\beta$-glycerolphosphate) for 15 or 21 days [28]. Medium was changed twice a week. After 2 weeks of osteogenic induction, the osteoblastic differentiation markers alkaline phosphatase (ALP) activity and type 1 collagen production were determined. Extracellular calcium was measured after 3 weeks of osteogenic induction.

\subsection{Determination of osteoblastic differentiation parameters}

ALP activity and type 1 collagen production was determined in cultures after 15 days in osteogenic medium. To determine ALP activity, cell monolayers were washed with phosphatebuffered saline (PBS) and then lysed with $200 \mu \mathrm{L}$ of $0.1 \%$ Triton-X100 solution. $100 \mu \mathrm{L}$ of the lysates were used to evaluate ALP activity by hydrolysis of p-nitrophenylphosphate (p-NPP) to p-nitrophenol (p-NP) at $37^{\circ} \mathrm{C}$. p-NP absorbance was recorded at $405 \mathrm{~nm}$ [34]. Protein determination was performed by the Bradford technique using aliquots of the same lysates [35]. To determine type 1 collagen production cell monolayers were fixed in Bouin's solution, stained with Sirius red dye for $1 \mathrm{~h}$ and stained material was then dissolved in $1 \mathrm{~mL} 0.1 \mathrm{~N} \mathrm{NaOH}$. The absorbance of the resulting solution was recorded at $550 \mathrm{~nm}$ [36]. After 21 days of osteogenic differentiation, extracellular calcium deposits (mineralization nodules) were measured using alizarin $\mathrm{S}$ red staining [37]. Stained calcium deposits were extracted with $1 \mathrm{~mL}$ of $0.1 \mathrm{~N}$ $\mathrm{NaOH}$ and the optical density was measured at $548 \mathrm{~nm}$.

\subsection{Statistical analysis}

Results are expressed as the mean \pm standard error of the mean (SEM) except for the pQCT analysis in which results are expressed as mean \pm standard deviation (SD). Differences between groups were assessed by one-way analysis of variance (ANOVA) followed by Tukey's post-test using InStat Graph Pad 3.0 software (Graph Pad Software, San Diego, CA, USA). Differences were considered significant when $p<0.05$.

\section{Results}

\subsection{Body weight and serum profiles}

Rats from the different experimental groups had comparable body weights at the end of the 5 -weeks treatment period $(284.0 \pm 14.3 \mathrm{~g}$ for $\mathrm{C}, 279.0 \pm 14.0 \mathrm{~g}$ for $\mathrm{CM}, 286.7 \pm 15.8 \mathrm{~g}$ for FRD, and $289.0 \pm 12.2 \mathrm{~g}$ for FRDM). Non-fasting serum profiles of the four experimental groups are shown in Table 1. Glucose levels were increased in FRD rats and these values were similar to control values after 2 weeks of metformin administration. CM group did not show alterations in glucose values,

\section{Table 1 - Non-fasting serum profiles after 5 weeks of treatment.}

\begin{tabular}{lllll} 
& C & CM & FRD & FRDM \\
\hline Glucose $[\mathrm{mg} / \mathrm{dL}]$ & $170.0 \pm 8.3$ & $160.8 \pm 8.6$ & $214.6 \pm 12.3^{*}, \#$ & $194.7 \pm 9.9$ \\
Insulin [ng/mL] & $1.18 \pm 0.75$ & $1.09 \pm 0.30$ & $5.89 \pm 0.98^{*}, \#$ & $4.02 \pm 3.10$ \\
HOMA-IR index & $2.34 \pm 0.68$ & $2.08 \pm 0.33$ & $15.00 \pm 0.22^{*}, \#$ & $9.28 \pm 0.82^{*}, \#,+$ \\
Triglycerides [mg/dL] & $70.0 \pm 6.1$ & $58.4 \pm 4.7$ & $130.1 \pm 7.8^{*}, \#$ & $115.8 \pm 7.3^{*}, \#$ \\
Fructosamine $[\mu \mathrm{mol} / \mathrm{L}]$ & $130 \pm 21$ & $190 \pm 13$ & $218 \pm 19^{*}$ & $239 \pm 16^{*}$ \\
Cholesterol $[\mathrm{mg} / \mathrm{dL}]$ & $46.9 \pm 1.5$ & $47.9 \pm 0.7$ & $51.1 \pm 1.4$ & $45.9 \pm 1.9$ \\
\hline
\end{tabular}

C: control group; CM: control plus oral metformin group; FRD: group of rats receiving 10\% fructose in drinking water; FRDM: group of rats receiving FRD plus metformin. HOMA-IR: Homeostasis Model Assessment-Insulin Resistance index. Differences: ${ }^{*} p<0.05$ vs C; ${ }^{*} p<0.05$ vs $\mathrm{CM} ;{ }^{\dagger} p<0.05$ vs FRD. 
which is consistent with the normoglycemic action of the drug. FRD rats also evidenced hyperinsulinemia and hypertriglyceridemia. Metformin administration did not change insulin values in $\mathrm{CM}$ compared with $\mathrm{C}$ rats. In contrast, insulinemia in FRDM group tended to be lower than in FRD rats, and this tendency was statistically significant when insulin resistance was estimated with the HOMA-IR index (Table 1). Furthermore, metformin slightly prevented the elevation of TG observed in FRD rats. Fructosamine levels were increased in FRD rats and metformin did not alter these levels (FRDM group, N.S. vs FRD). No significant differences were observed in cholesterol levels. These results indicate that the fructose rich diet induced carbohydrate and lipid metabolism alterations resembling human MS, and that the administration of metformin for three weeks tended to prevent these changes.

\subsection{Bone length analysis}

Femoral length was comparable among rats of the four experimental groups $(32.58 \pm 0.19 \mathrm{~mm}$ for C, $32.20 \pm 0.19 \mathrm{~mm}$ for $\mathrm{CM}, 32.12 \pm 0.21 \mathrm{~mm}$ for FRD, and $32.20 \pm 0.16 \mathrm{~mm}$ for FRDM). The same result was observed with tibial lengths (37.61 $\pm 0.27 \mathrm{~mm}$ for $\mathrm{C}, 37.03 \pm 0.08 \mathrm{~mm}$ for $\mathrm{CM}, 36.99 \pm 0.25 \mathrm{~mm}$ for
FRD, and $37.50 \pm 0.23 \mathrm{~mm}$ for FRDM). Similar results were found for the length of the body of the first lumbar vertebrae $(6.09 \pm 0.02 \mathrm{~mm}$ for $\mathrm{C}, 6.51 \pm 0.26 \mathrm{~mm}$ for $\mathrm{CM}, 6.43 \pm 0.10 \mathrm{~mm}$ for $\mathrm{FRD}$, and $6.30 \pm 0.19 \mathrm{~mm}$ for $\mathrm{FRDM}$ ).

\section{3. $\quad$ QCT analysis of trabecular and cortical bone}

Trabecular bone parameters assessed in distal femoral metaphyses and in proximal tibial metaphyses showed no significant differences among groups (Table 2). Similar results were found in the body of the second lumbar vertebrae (data not shown). In addition, parameters of cortical bone in femoral and tibial midshaft showed similar values in all experimental groups (Table 3).

\subsection{Structural and static histomorphometric analyses of distal femoral metaphysis}

The evaluation of structural parameters of trabecular bone present in the distal metaphysis showed similar values for bone volume (BV/TV; Fig. 2, panel A), number of trabeculae, trabecular width and trabecular separation among the four experimental groups (Fig. 2, panels B-D).

\begin{tabular}{|c|c|c|c|c|}
\hline Distal femoral metaphysis & C & $\mathrm{CM}$ & FRD & FRDM \\
\hline Total BMC [mg/cm] & $11.31 \pm 1.46$ & $10.34 \pm 0.94$ & $10.35 \pm 1.07$ & $10.71 \pm 0.72$ \\
\hline Total BMD $\left[\mathrm{mg} / \mathrm{cm}^{3}\right]$ & $660.30 \pm 57.74$ & $625.25 \pm 27.42$ & $645.68 \pm 10.35$ & $659.45 \pm 18.28$ \\
\hline Trabecular BMC [mg/cm] & $1.71 \pm 0.48$ & $1.42 \pm 0.33$ & $1.47 \pm 0.40$ & $1.48 \pm 0.22$ \\
\hline Trabecular BMD $\left[\mathrm{mg} / \mathrm{cm}^{3}\right]$ & $331.10 \pm 79.28$ & $284.45 \pm 48.80$ & $302.83 \pm 53.11$ & $303.80 \pm 33.93$ \\
\hline Proximal tibial metaphysis & C & CM & FRD & FRDM \\
\hline Total BMC [mg/cm] & $7.29 \pm 0.38$ & $7.05 \pm 0.57$ & $7.26 \pm 0.51$ & $7.31 \pm 0.40$ \\
\hline Total BMD $\left[\mathrm{mg} / \mathrm{cm}^{3}\right]$ & $629.83 \pm 21.51$ & $593.30 \pm 12.11$ & $630.33 \pm 13.18$ & $616.98 \pm 27.38$ \\
\hline Trabecular BMC [mg/cm] & $0.71 \pm 0.14$ & $0.61 \pm 0.08$ & $0.62 \pm 0.14$ & $0.60 \pm 0.13$ \\
\hline Trabecular BMD $\left[\mathrm{mg} / \mathrm{cm}^{3}\right]$ & $202.70 \pm 37.80$ & $169.03 \pm 20.44$ & $179.18 \pm 24.58$ & $166.35 \pm 24.65$ \\
\hline
\end{tabular}

C: control group; CM: control plus oral metformin group; FRD: group of rats receiving 10\% fructose in drinking water; FRDM: group of rats receiving FRD plus metformin. BMC: bone mineral content; BMD: bone mineral density; results are expressed as the media \pm SD.

\section{Table 3 - pQCT analysis of femoral and tibial midshaft to evaluate cortical bone status.}

\begin{tabular}{lllll} 
Femoral midshaft & C & CM & FRD & FRDM \\
\hline Cortical BMC [mg/cm] & $8.15 \pm 0.24$ & $8.03 \pm 0.34$ & $8.06 \pm 0.59$ & $8.12 \pm 0.74$ \\
Cortical BMD [mg/cm $\left.{ }^{3}\right]$ & $1233.85 \pm 14.19$ & $1223.33 \pm 26.35$ & $1247.03 \pm 11.32$ & $1252.03 \pm 14.42$ \\
Cortical Thickness [mm] & $0.76 \pm 0.02$ & $0.76 \pm 0.01$ & $0.75 \pm 0.04$ & $0.76 \pm 0.03$ \\
Periosteal circumference [mm] & $11.05 \pm 0.15$ & $11.05 \pm 0.31$ & $10.96 \pm 0.38$ & $10.93 \pm 0.55$ \\
Endocortical circumference [mm] & $6.26 \pm 0.14$ & $6.29 \pm 0.31$ & $6.24 \pm 0.29$ & $6.18 \pm 0.42$ \\
\hline Tibial midshaft & $\mathrm{C}$ & $\mathrm{CM}$ & FRD & FRDM \\
\hline Cortical BMC [mg/cm] & $6.22 \pm 0.18$ & $6.14 \pm 0.29$ & $6.27 \pm 0.57$ & $6.28 \pm 0.55$ \\
Cortical BMD [mg/cm $\left.{ }^{3}\right]$ & $1215.68 \pm 10.23$ & $1206.60 \pm 8.53$ & $1216.95 \pm 12.99$ & $1206.00 \pm 11.56$ \\
Cortical Thickness [mm] & $0.81 \pm 0.02$ & $0.80 \pm 0.03$ & $0.83 \pm 0.07$ & $0.82 \pm 0.04$ \\
Periosteal circumference [mm] & $8.88 \pm 0.05$ & $8.89 \pm 0.19$ & $8.82 \pm 0.34$ & $8.91 \pm 0.35$ \\
Endocortical circumference [mm] & $3.80 \pm 0.09$ & $3.88 \pm 0.17$ & $3.61 \pm 0.19$ & $3.76 \pm 0.18$ \\
\hline
\end{tabular}

C: control group; CM: control plus oral metformin group; FRD: group of rats receiving 10\% fructose in drinking water; FRDM: group of rats receiving FRD plus metformin. Results are expressed as the media \pm SD. 

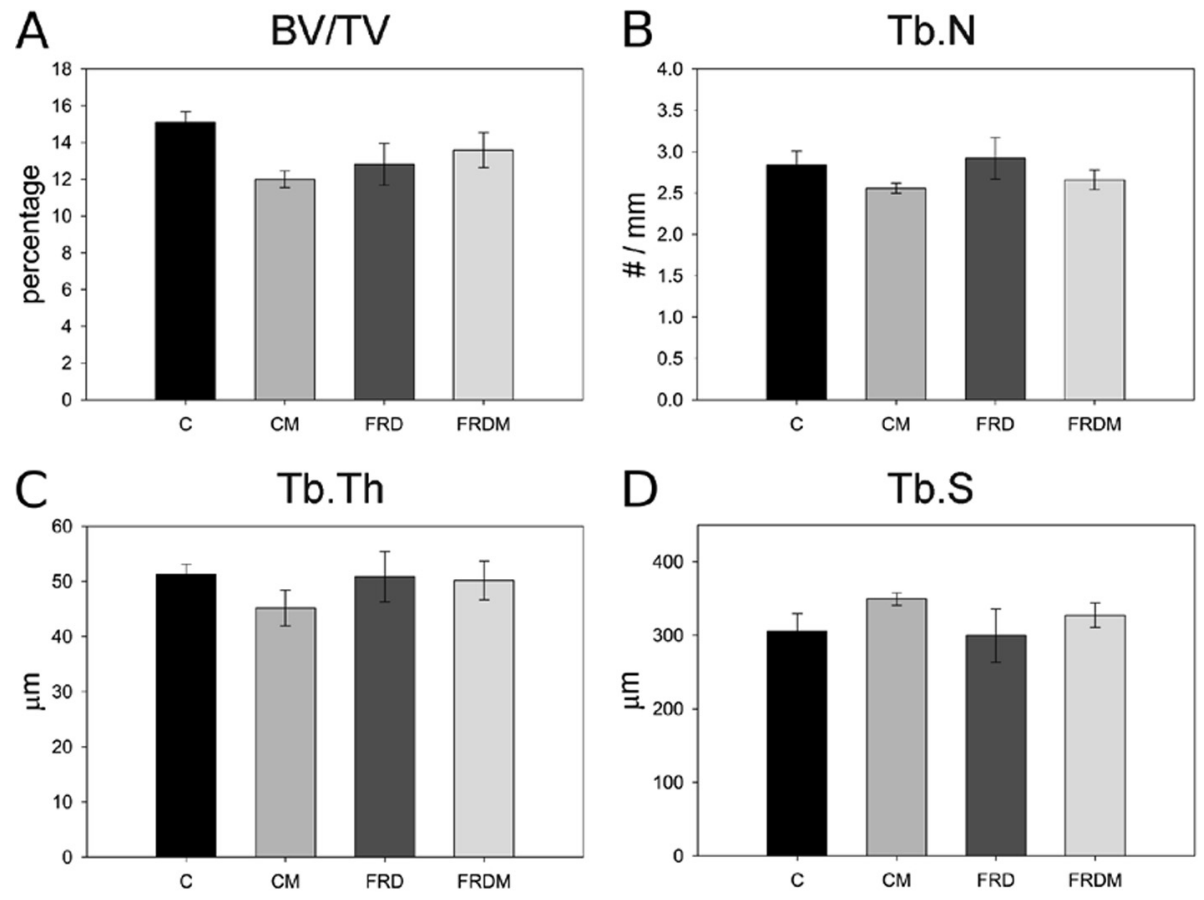

Fig. 2 - Structural histomorphometric analysis of distal femoral metaphysis. C: control group; CM: control plus oral metformin group; FRD: group of rats receiving $10 \%$ fructose in drinking water; FRDM: group of rats receiving FRD plus metformin.

Analysis of trabecular bone structure parameters, including: (A) trabecular bone volume, BV/TV; (B) trabecular number, Tb.N; (C) trabecular thickness, Tb.Th; and (D) trabecular separation, Tb.S.
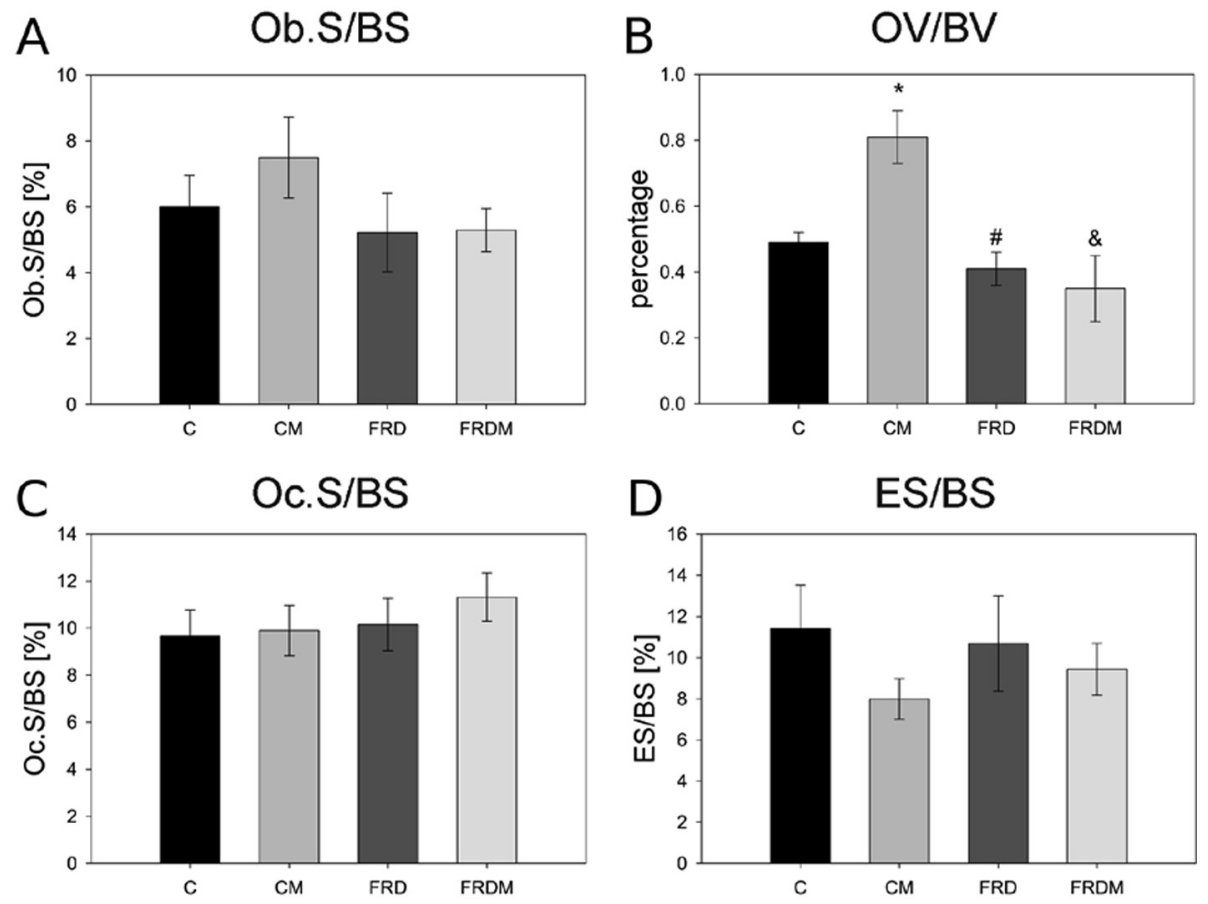

Fig. 3 - Static histomorphometric analysis of distal femoral metaphysis. C: control group; CM: control plus oral metformin group; FRD: group of rats receiving $10 \%$ fructose in drinking water; FRDM: group of rats receiving FRD plus metformin. Analysis of trabecular bone cells parameters including: (A) osteoblast surface, Ob.S; (B) osteoid volume, OV/BV; (C) osteoclast surface, Oc.S; and (D) eroded surface, ES/BS. Differences: ${ }^{*} p<0.05$ vs C, ${ }^{\#} p<0.05$ vs CM, ${ }^{\&} p<0.01$ vs CM.

Fig. 3 shows the results of the static histomorphometric analysis. FRD and FRDM groups had a trend for a lower, although not statistically different, osteoblast surface and osteoid volume compared to rats of group C (Fig. 3, panels A and $\mathrm{B}$ ). CM rats had higher values for these parameters compared to group C. Osteoclast surface (Oc.S) and eroded surface 

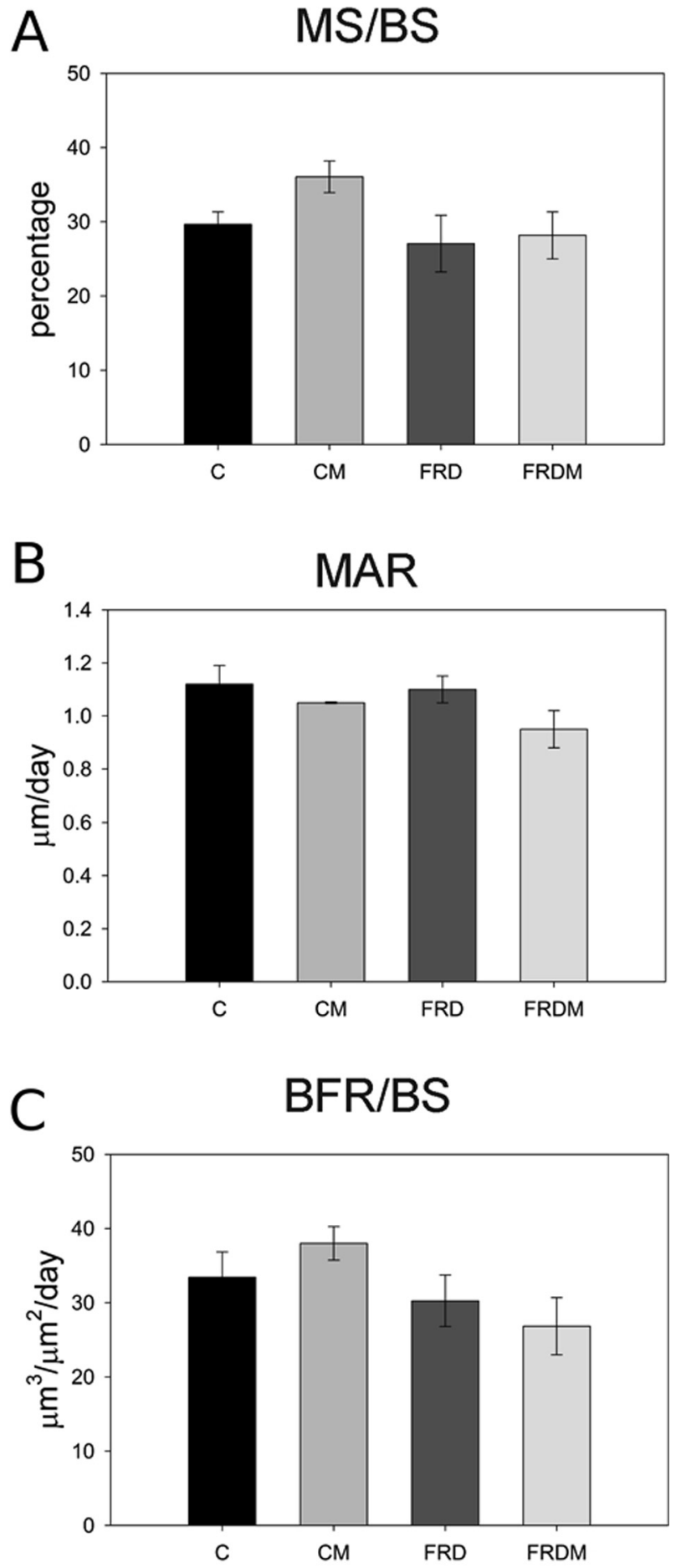

Fig. 4 - Dynamic histomorphometric analysis of distal femoral metaphysis. C: control group; CM: control plus oral metformin group; FRD: group of rats receiving $10 \%$ fructose in drinking water; FRDM: group of rats receiving FRD plus metformin. Fluorochrome-based indices of bone formation were measured in the trabecular bone from the distal femoral metaphysis: (A) mineralizing surface, MS/BS; (B) mineral apposition rate, MAR; and (C) bone formation rate, BFR/BS.
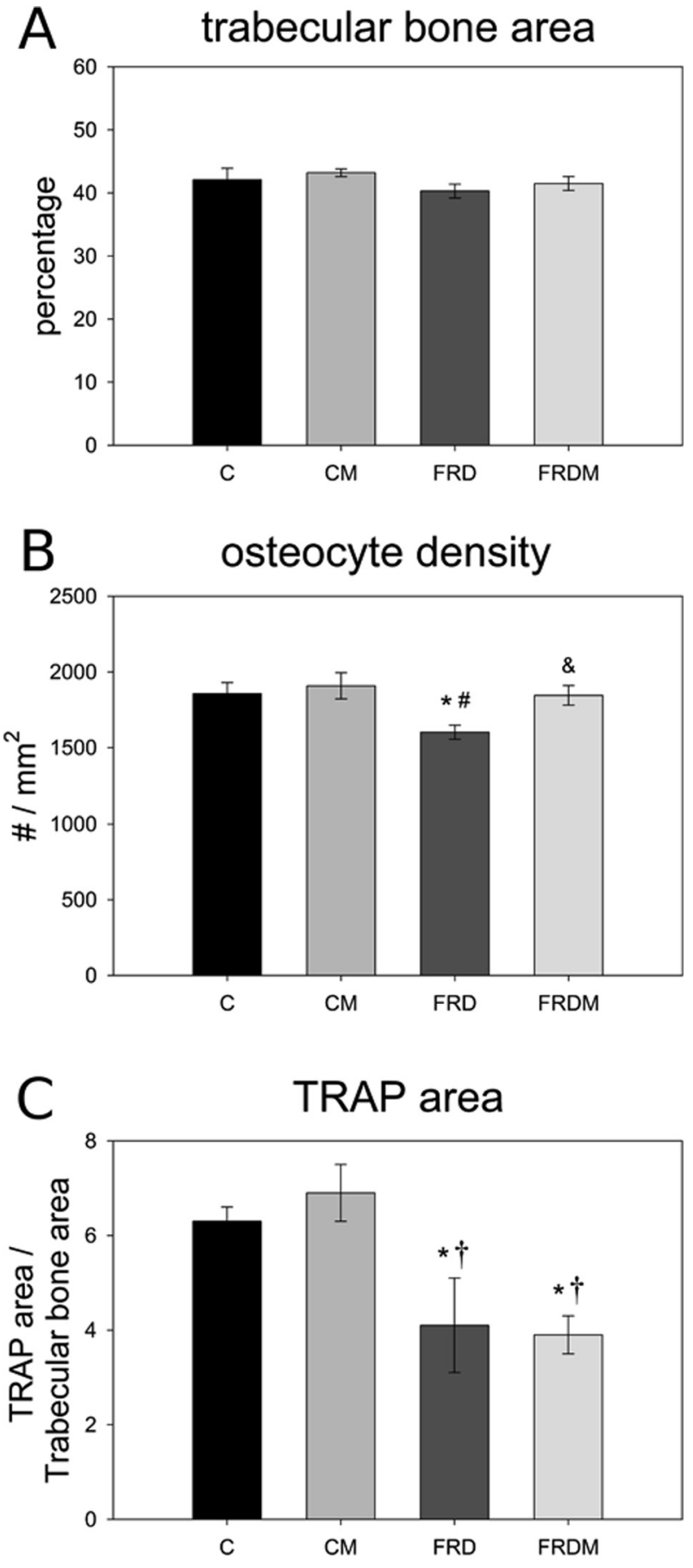

Fig. 5 - Static histomorphometric analysis of proximal femoral metaphysis. C: control group; CM: control plus oral metformin group; FRD: group of rats receiving $10 \%$ fructose in drinking water; FRDM: group of rats receiving FRD plus metformin. Analysis of trabecular bone parameters including: (A) trabecular bone area, (B) osteocyte density, and (C) TRAP area in the primary spongiosa. Differences: ${ }^{*} p<0.05$ vs $C,{ }^{\dagger} p<0.05$ vs CM, ${ }^{\#} p<0.01$ vs CM, ${ }^{\circledR} p<0.01$ vs FRD. 


\section{A alkaline phosphatase activity}

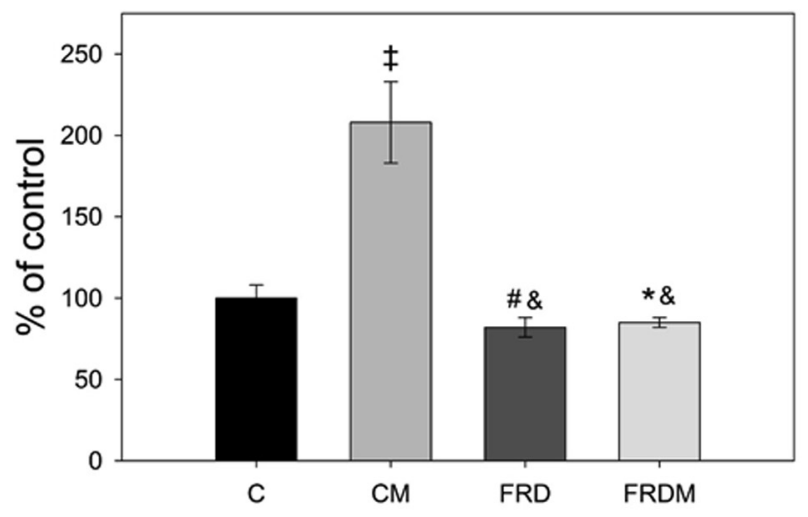

\section{B type 1 collagen production}

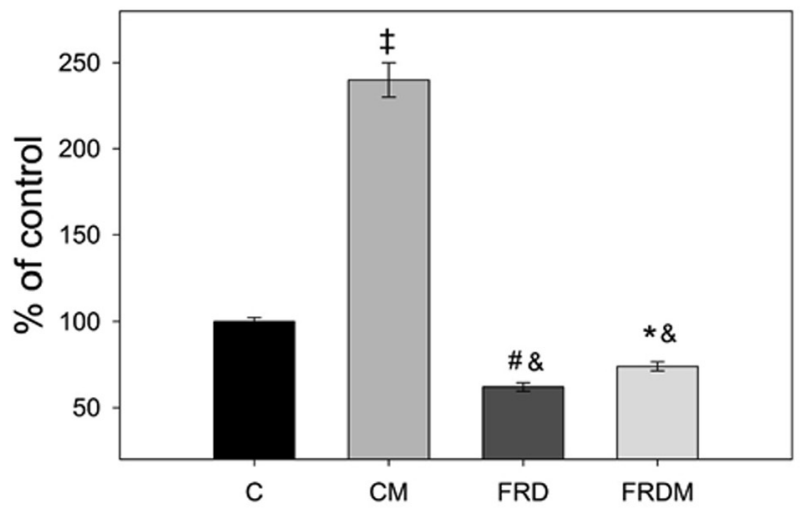

\section{C extracellular calcium deposits}

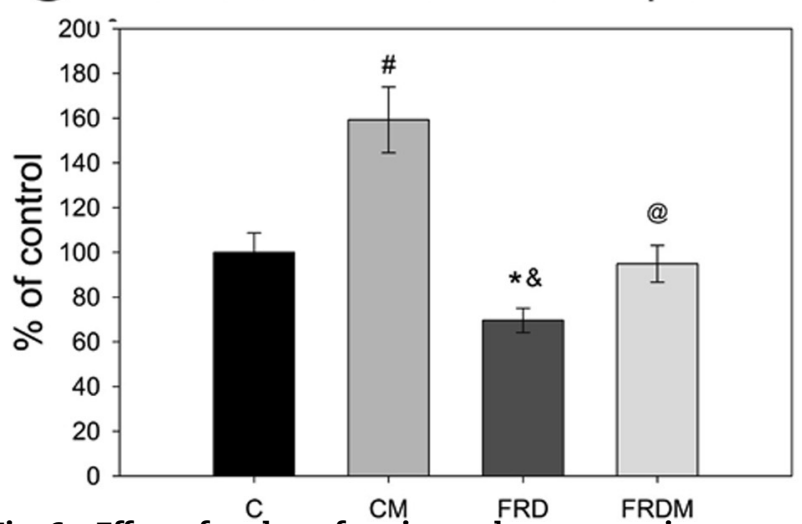

Fig. 6 - Effect of oral metformin on the osteogenic differentiation potential of MSC from rats with fructoseinduced MS. C: control group; CM: control plus oral metformin group; FRD: group of rats receiving $10 \%$ fructose in drinking water; FRDM: group of rats receiving FRD plus metformin. MSC obtained from the femora of the four experimental groups were cultured in an osteogenic medium, and then evaluated for alkaline phosphatase (ALP) activity (A) and type 1 collagen production (B) after 15 days of differentiation, and formation of extracellular nodules of mineralization (C) after 21 days of differentiation. Differences: ${ }^{p} p<0.05$ vs C, ${ }^{\#} p<0.01$ vs C, ${ }^{\ddagger} p<0.001$ vs C, ${ }^{\circledR} p<0.01$ vs $\mathrm{CM},{ }^{{ }^{8}} p<0.001$ vs $\mathrm{CM}$.
(ES/BS) were not significantly different among the different experimental groups (Fig. 3, panels C and D).

3.5. Dynamic histomorphometric analysis of distal femoral metaphysis

Dynamic histomorphometric analysis revealed that MS/BS, MAR and BFR/BS were not significantly affected by treatments (Fig. 4, panels A-C). In addition, there were no statistically significant differences in femoral longitudinal bone growth among rats of the different experimental groups $(29.45$ $\pm 0.74 \mu \mathrm{m} /$ day for C, $28.91 \pm 0.90 \mu \mathrm{m} /$ day for $C M, 27.14$ $\pm 2.51 \mu \mathrm{m} /$ day for FRD, and $28.64 \pm 1.24 \mu \mathrm{m} /$ day for FRDM).

3.6. Static histomorphometric analysis of proximal femoral metaphysis

The analysis of relative trabecular bone area revealed that although there were no significant differences among groups, FRD rats tended to show lower values for this parameter (Fig. 5, panel A). In addition, osteocytic density of trabecular bone was significantly decreased in FRD rats ( $86 \%$ of C). However, metformin administration to rats on a fructose rich diet (FRDM group) restored the values of osteocyte density to those of the control group (Fig. 5, panel B). Analysis of TRAP stained bone sections showed that osteoclastic density was decreased in the primary spongiosa of FRD rats, and that oral metformin was unable to restore these values to control levels (Fig. 5, panel C). In rats from group CM, all three parameters showed a trend for larger values, although not statistically different, compared to rats of the $C$ group.

\subsection{Osteogenic potential of MSC}

After 15 days of osteogenic induction, MSCs derived from FRD rats expressed lower levels of ALP and produced lower amounts of type 1 collagen than MSC obtained from $C$ rats ( $72 \%$ and $62 \%$ of C, respectively) (Fig. 6, panels A and B). Similarly, after 21 days of osteogenic induction, a significant decrease in formation of mineralization nodules was observed in MSC isolated from the FRD group (68\% of C) (Fig. 6, panel C). Metformin administration (FRDM group) did not modify ALP activity and type 1 collagen production of MSC, although it completely prevented the fructose-induced decrease in extracellular mineralization.

\section{Discussion}

Over the past decades, the prevalence of conditions such as obesity, MS and Diabetes mellitus has increased dramatically. In particular, MS has become an epidemic due to an increase in obesity and sedentary lifestyles [38].

Animals fed fructose rich diets (FRD) are widely used as models of MS, and reflect the effect of environmental factors on the development of MS. In these models animals exhibit a metabolic condition that resembles human MS, showing insulin resistance, dyslipidemia, increased blood pressure, impaired glucose tolerance and, in some cases, obesity $[10,18,21-25]$. We have recently demonstrated that 
FRD also induces alterations in bone metabolism, decreasing the osteogenic potential of mesenchymal stem cells (MSC) [9].

\subsection{Effects of metformin on glucose and lipid metabolism in the FRD model of MS}

Metformin is an insulin-sensitizing biguanide that is widely administered to patients with type 2 Diabetes mellitus and/ or MS. It inhibits hepatic glucose production and it may also improve glucose uptake by peripheral tissues [39]. In the present study, metformin administration in drinking water showed slight, although no statistically significant, beneficial effects on the alterations observed in serum glucose, insulin and triglycerides of FRD rats (Table 1). Nevertheless, metformin induced a significant decrease in the HOMA-index of fructose-fed rats, suggesting an improvement in the insulin resistance of FDR animals. However, no changes were observed in fructosamine levels, indicating that in our experimental conditions metformin administration does not have a beneficial effect in controlling the increase in non-enzymatic glycosylation of serum proteins induced by FRD that has been previously described by other authors [12,40].

\subsection{Effects of metformin on bone tissue in the FRD model of MS}

In our previous studies we demonstrated that the addition of $10 \% \mathrm{w} / \mathrm{v}$ fructose to the drinking water of rats for four weeks induced deleterious alterations in bone microarchitecture and in the regeneration of bone lesions [9].

Metformin has been shown to produce beneficial effects on certain features of the MS (such as ROS increase) that negatively correlate with bone quality [41,42]. Oral administration of metformin has also been associated with increased osteogenesis in vivo and in vitro $[15,16]$. Hence, this drug appears to improve bone cellular machinery by acting directly on osteoblasts $[15,43]$ or indirectly by improving characteristics that negatively affect bone. For example, metformin prevents the formation of reactive oxygen species (ROS) and this could avoid the alterations caused by oxidative stress on different tissues and cells, including osteoblasts [44-46].

Bone was evaluated histomorphometrically, measuring the number and density of trabeculae as well as the status of cells that contribute to maintain them; and at the level of mesenchymal stromal cells (MSCs) analyzing their osteogenic potential. Confirming our previous findings [9], the results of this study show that the group of rats which received fructose and metformin (FRDM group) had similar osteocytic density compared to control rats, indicating that metformin can prevent the alterations in osteocyte density observed in FRD group. Although we have not evaluated mechanisms of cell death in this study, the decrease in osteocyte density of FRD animals could be due to increased apoptosis, an effect that could be prevented by metformin. We have previously shown that metformin can prevent the apoptosis induced by AGEs on cultured osteoblasts [45]. CM rats showed higher values for these parameters than $C$ rats, although the differences were not significantly different (Fig. 5). A reduction in the osteocyte network could lead to trabecular bone with a decreased response to mechanotransduction and/or impaired ability to repair perilacunar microdamage $[47,48]$. In vivo studies have shown that metformin improves bone microarchitecture in diabetic rats $[16,49]$, although another study showed that metformin was unable to prevent bone loss in an ovariectomy model of osteoporosis [50]. Thus, metformin should not be considered an anti-osteoporotic drug but rather an insulin-sensitizer that may secondarily improve bone metabolism.

The PQCT analysis of trabecular bone in distal femoral and proximal tibial metaphyses showed no alterations in the FRD group (Table 2). The groups of rats that received metformin (CM and FRDM) did not increase BMC and BMD in cancellous bone when compared with groups that did not receive the drug ( $C$ and FRD respectively). These results are consistent with histomorphometric data of distal femoral metaphysis (Fig. 3). The structural parameters (trabecular bone volume, trabecular number, trabecular thickness, and trabecular separation) observed at this anatomic bone location showed similar values in the four experimental groups. These data together suggest that the duration of this study was not sufficiently long enough to induce histologic bone alterations in vivo. Nevertheless, lack of changes in BMC or BMD does not imply absence of increased bone fragility and/or bone alterations. Even though BMD is usually used to estimate fracture risk, it is not always a good predictor because it is related to bone quantity rather that to its quality. For example, patients with type 2 Diabetes mellitus show normal or elevated $\mathrm{BMD}$, they also have elevated rates of osteoporotic fractures [51].

In some conditions, such as MS, Diabetes mellitus and aging, it is frequent to observe formation of advanced glycation end-products (AGEs) by non-enzymatic glycosylation. This reaction occurs between amino groups present in proteins, lipids or nucleic acids and reducing sugars or carbonylic intermediates. Proteins with long half-lives, such as type 1 collagen (which is the principal component of bone tissue extracellular matrix), are prone to suffer this reaction, particularly in conditions associated with chronically elevated plasma glucose levels. This process leads to formation of pathologic cross-links, which ultimately induce loss of flexibility and elasticity in bone tissue and increased bone fragility. In addition, excess AGEs alters bone cell homeostasis via their binding to specific plasma membrane receptors, leading to decreased osteoblast and osteoclast functions and eventually decreased bone turnover and bone tissue quality $[12,14]$.

Osteoblast surface and osteoid volume are two important parameters of bone formation [49]. The analysis of these parameters in distal femoral metaphysis showed lower values for FRD group, although the difference was not statistically significant (Fig. 3). Metformin administration to FRD rats (FRDM group) did not improve these parameters, although $\mathrm{CM}$ rats had increased osteoid volume. Osteoid synthesis is the first step in bone formation, leading to mature bone after its mineralization. Bone resorption-related parameters (osteoclast and eroded surfaces) showed no significant differences among experimental groups (Fig. 3). These results sug- 
gest that the period of time of metformin administration was not enough to normalize the mild alterations observed in bone remodeling parameters in rats of the FRD group. Osteoblasts and osteoclasts act in a coordinate fashion during bone remodeling. Remodeling cycles, where a normal osteoclastic activity is followed by reduced osteoblast activity for longer periods of time than those observed in this study, could lead to negative bone balance and promoting skeletal bone loss.

Dynamic histomorphometric parameters of the distal femur were similar in the four experimental groups (Fig. 4), showing no differences in the bone formation rate or in longitudinal bone growth. This is in agreement with the similarities between bone lengths across all experimental groups.

\subsection{Effects of metformin on mesenchymal stromal cells}

Mesenchymal stromal cells (MSC) are the precursors of all the cells present in bone tissue, except osteoclasts. Modifications in the bone marrow microenvironment could lead to a shift in the differentiation potential towards different cell lines. In MS, the presence of ROS, proinflammatory cytokines, or AGEs could reduce MSC osteogenic potential and predispose the cells to other phenotypes. We have previously demonstrated that fructose induced MS is associated with a deviation in the adipogenic/osteogenic commitment of MSC, probably by modulation of the Runx2/PPARgamma ratio, but no differences in bone marrow adiposity were seen in this condition when compared with control rats [9].

It has been reported that metformin has direct ex vivo effects on MSCs, predisposing these cells to differentiate into the osteoblastic phenotype instead of into the adipocytic lineage [28]. Similar effects have been seen in MSCs isolated from rats with partial insulin deficiency [16]. This may lead to a higher number of osteoblasts and therefore, to an increased bone formation. In the present study although metformin treatment of control animals greatly increased the osteogenic induction of MSC (for all parameters evaluated), in FRD animals this effect of metformin was less clear cut. Metformin administration (FRDM group) did not modify ALP activity or type 1 collagen production in MSC, although it completely prevented the fructose-induced decrease in extracellular mineralization. It is possible that the incomplete preventive effects of metformin observed in this study could be as a result of the relatively short treatment period used in this study.

\subsection{About the election of treatment time period}

The insulin resistance present in this model produces hyperglycemia. This produces pancreatic $\beta$ cells hyperstimulation as a compensatory mechanism to produce higher amounts of insulin and try to reduce glycemic values (compensatory hyperglycemia). If this condition was sustained in time, it could lead to $\beta$ cell exhaustion with a consequent decrease in the functionality or amount of these cells and Diabetes development [52]. This scenario would change the perspectives of this study because we would not be studying a model with insulin resistance, but another one with partial reduction in the secretion of this hormone.
In conclusion, our model of FRD-induced MS shows a reduction in MSC osteogenic induction, in osteocyte density and in TRAP activity. Thus, FRD appears to induce a decrease in bone remodeling. Although in our experimental conditions oral treatment with metformin did not improve all bone structural alterations induced by FRD, it was able to prevent trabecular osteocyte loss and the decreased extracellular mineralization induced by FRD on MSCs.

\section{Funding}

The project described was supported by PICT 0053-2012 grant from Agencia to A.D.M.; grants from Universidad Nacional de La Plata and Comisión de Investigaciones Científicas de la Provincia de Buenos Aires (CICPBA) to A.M.C. J.I.F. received funds from Universidad Nacional de La Plata.

\section{Disclosure}

The authors declare no conflict of interest.

\section{Acknowledgements}

We thank Montpelier Laboratories for the provision of metformin. J.I.F. is a fellowship from CONICET and A.M.C. is a member of the Research Career from CICPBA. We thank Dr. Thomas J. Wronski for providing advices and support for the histomorphometry analysis.

\section{R E F E R E N C E S}

[1] Lam DW, LeRoith D. Metabolic syndrome. In: De Groot LJ, Beck-Peccoz P, Chrousos G, Dungan K, Grossman A, Hershman JM, et al., editors. Endotext. South Dartmouth MA: MDText.com, Inc.; 2000.

[2] Reaven GM. Banting lecture 1988. Role of insulin resistance in human disease. Diabetes 1988;1988 (37):1595-607.

[3] Alberti KG, Eckel RH, Grundy SM, Zimmet PZ, Cleeman JI, Donato KA, et al. Harmonizing the metabolic syndrome: a joint interim statement of the international diabetes federation task force on epidemiology and prevention; national heart, lung, and blood institute; american heart association; world heart federation; international atherosclerosis society; and international association for the study of obesity. Circulation 2009;120:1640-5.

[4] Leslie WD, Rubin MR, Schwartz AV, Kanis JA. Type 2 diabetes and bone. J Bone Miner Res 2012;27:2231-7.

[5] Oei L, Zillikens MC, Dehghan A, Buitendijk GH, CastanoBetancourt MC, Estrada K, et al. High bone mineral density and fracture risk in type 2 diabetes as skeletal complications of inadequate glucose control: the rotterdam study. Diabetes Care 2013;36:1619-28.

[6] El Maghraoui A, Rezqi A, El Mrahi S, Sadni S, Ghozlani I, Mounach A. Osteoporosis, vertebral fractures and metabolic syndrome in postmenopausal women. BMC Endocr Disord 2014;14:93.

[7] Sun M, Cao M, Fu Q, Zhu Z, Meng C, Mao J, et al. Association of calcaneal quantitative ultrasound parameters with metabolic syndrome in middle-aged and elderly Chinese: a 
large population-based cross-sectional study. BMC Endocr Disord 2014;14:14.

[8] Panchal SK, Brown L. Rodent models for metabolic syndrome research. J Biomed Biotechnol 2011;2011:351982.

[9] Felice JI, Gangoiti MV, Molinuevo MS, McCarthy AD, Cortizo AM. Effects of a metabolic syndrome induced by a fructoserich diet on bone metabolism in rats. Metab Clin Exp 2014;63:296-305.

[10] Hwang IS, Ho H, Hoffman BB, Reaven GM. Fructose-induced insulin resistance and hypertension in rats. Hypertension 1987;10:512-6 (Dallas, Tex: 1979).

[11] Mamikutty N, Thent ZC, Sapri SR, Sahruddin NN, Mohd Yusof MR, Haji Suhaimi F. The establishment of metabolic syndrome model by induction of fructose drinking water in male Wistar rats. Biomed Res Int 2014;2014:263897.

[12] Mikulikova K, Eckhardt A, Kunes J, Zicha J, Miksik I. Advanced glycation end-product pentosidine accumulates in various tissues of rats with high fructose intake. Physiol Res 2008;57:89-94.

[13] Franke S, Ruster C, Pester J, Hofmann G, Oelzner P, Wolf G. Advanced glycation end products affect growth and function of osteoblasts. Clin Exp Rheumatol 2011;29:650-60.

[14] McCarthy AD, Molinuevo MS, Cortizo AM. AGEs and bone ageing in diabetes mellitus. J Diabetes Metab 2013.

[15] Cortizo AM, Sedlinsky C, McCarthy AD, Blanco A, Schurman L. Osteogenic actions of the anti-diabetic drug metformin on osteoblasts in culture. Eur J Pharmacol 2006;536:38-46.

[16] Tolosa MJ, Chuguransky SR, Sedlinsky C, Schurman L, McCarthy AD, Molinuevo MS, et al. Insulin-deficient diabetesinduced bone microarchitecture alterations are associated with a decrease in the osteogenic potential of bone marrow progenitor cells: preventive effects of metformin. Diabetes Res Clin Pract 2013;101:177-86.

[17] UFAW. The UFAW handbook on the care and management of laboratory and other research animals. 8th ed. United Kingdom: Wiley-Blackwell; 2011.

[18] Peredo HA, Mayer MA, Rodriguez Fermepin M, Grinspon D, Puyo AM. Oral treatment and in vitro incubation with fructose modify vascular prostanoid production in the rat. Auton Autacoid Pharmacol 2006;26:15-20.

[19] Rebolledo OR, Marra CA, Raschia A, Rodriguez S, Gagliardino JJ. Abdominal adipose tissue: early metabolic dysfunction associated to insulin resistance and oxidative stress induced by an unbalanced diet. Horm Metab Res 2008;40:794-800.

[20] Basciano H, Federico L, Adeli K. Fructose, insulin resistance, and metabolic dyslipidemia. Nutr Metab 2005;2:5.

[21] Bezerra RM, Ueno M, Silva MS, Tavares DQ, Carvalho CR, Saad MJ. A high fructose diet affects the early steps of insulin action in muscle and liver of rats. J Nutr 2000;130:1531-5.

[22] Maiztegui B, Borelli MI, Raschia MA, Del Zotto H, Gagliardino $\mathrm{JJ}$. Islet adaptive changes to fructose-induced insulin resistance: beta-cell mass, glucokinase, glucose metabolism, and insulin secretion. J Endocrinol 2009;200:139-49.

[23] Oron-Herman M, Kamari Y, Grossman E, Yeger G, Peleg E, Shabtay Z, et al. Metabolic syndrome: comparison of the two commonly used animal models. Am J Hypertens 2008;21:1018-22.

[24] Thorburn AW, Storlien LH, Jenkins AB, Khouri S, Kraegen EW. Fructose-induced in vivo insulin resistance and elevated plasma triglyceride levels in rats. Am J Clin Nutr 1989;49:1155-63.

[25] Thresher JS, Podolin DA, Wei Y, Mazzeo RS, Pagliassotti MJ. Comparison of the effects of sucrose and fructose on insulin action and glucose tolerance. Am J Physiol Regul Integr Comp Physiol 2000;279:R1334-40.

[26] Guidance for industry estimating the maximum safe starting dose in initial clinical trials for therapeutics in adult healthy volunteers. U.S. Department of Health and Human Services,
Food and Drug Administration, Center for Drug Evaluation and Research (CDER); 2005.

[27] Choi YH, Lee MG, Lee I. Effects of diabetes mellitus induced by alloxan on the pharmacokinetics of metformin in rats: restoration of pharmacokinetic parameters to the control state by insulin treatment. J Pharm Pharm Sci 2008;11:88-103.

[28] Molinuevo MS, Schurman L, McCarthy AD, Cortizo AM, Tolosa MJ, Gangoiti MV, et al. Effect of metformin on bone marrow progenitor cell differentiation: in vivo and in vitro studies. J Bone Miner Res 2010;25:211-21.

[29] Aguirre JI, Franz SE, Altman MK, Stabley JN, Lin X, Zamora PO, et al. Skeletal effects of fibroblast growth factor mimetic (F2A) in ovariectomized rats. J Musculoskelet Neuronal Interact 2009;9:38-43.

[30] Cacho J, Sevillano J, de Castro J, Herrera E, Ramos MP. Validation of simple indexes to assess insulin sensitivity during pregnancy in Wistar and Sprague-Dawley rats. Am J Physiol Endocrinol Metab 2008;295:E1269-76.

[31] Bemenderfer TB, Harris JS, Condon KW, Kacena MA. Tips and techniques for processing and sectioning undecalcified murine bone specimens. Methods Mol Biol 2014;1130:123-47.

[32] Parfitt AM, Drezner MK, Glorieux FH, Kanis JA, Malluche H, Meunier PJ, et al. Bone histomorphometry: standardization of nomenclature, symbols, and units. Report of the ASBMR histomorphometry nomenclature committee. J Bone Miner Res 1987;2:595-610.

[33] Tropel P, Noel D, Platet N, Legrand P, Benabid AL, Berger F. Isolation and characterisation of mesenchymal stem cells from adult mouse bone marrow. Exp Cell Res 2004;295:395-406.

[34] Cortizo AM, Etcheverry SB. Vanadium derivatives act as growth factor-mimetic compounds upon differentiation and proliferation of osteoblast-like UMR106 cells. Mol Cell Biochem 1995;145:97-102.

[35] Bradford MM. A rapid and sensitive method for the quantitation of microgram quantities of protein utilizing the principle of protein-dye binding. Anal Biochem 1976;72:248-54.

[36] Tullberg-Reinert H, Jundt G. In situ measurement of collagen synthesis by human bone cells with a sirius red-based colorimetric microassay: effects of transforming growth factor beta2 and ascorbic acid 2-phosphate. Histochem Cell Biol 1999;112:271-6.

[37] Ueno A, Kitase Y, Moriyama K, Inoue H. MC3T3-E1conditioned medium-induced mineralization by clonal rat dental pulp cells. Matrix Biol 2001;20:347-55.

[38] Yamaoka K, Tango T. Effects of lifestyle modification on metabolic syndrome: a systematic review and meta-analysis. BMC Med 2012;10:138.

[39] Gong L, Goswami S, Giacomini KM, Altman RB, Klein TE. Metformin pathways: pharmacokinetics and pharmacodynamics. Pharmacogenet Genomics 2012;22:820-7.

[40] Schalkwijk CG, Stehouwer CD, van Hinsbergh VW. Fructosemediated non-enzymatic glycation: sweet coupling or bad modification. Diabetes Metab Res Rev 2004;20:369-82.

[41] Harden KA, Cowan PA, Velasquez-Mieyer P, Patton SB. Effects of lifestyle intervention and metformin on weight management and markers of metabolic syndrome in obese adolescents. J Am Acad Nurse Pract 2007;19:368-77.

[42] Miranda PJ, DeFronzo RA, Califf RM, Guyton JR. Metabolic syndrome: evaluation of pathological and therapeutic outcomes. Am Heart J 2005;149:20-32.

[43] Jang WG, Kim EJ, Bae IH, Lee KN, Kim YD, Kim DK, et al. Metformin induces osteoblast differentiation via orphan nuclear receptor SHP-mediated transactivation of Runx2. Bone 2011;48:885-93. 
[44] Erejuwa OO, Sulaiman SA, Wahab MS, Salam SK, Salleh MS, Gurtu S. Comparison of antioxidant effects of honey, glibenclamide, metformin, and their combinations in the kidneys of streptozotocin-induced diabetic rats. Int J Mol Sci 2011;12:829-43.

[45] Schurman L, McCarthy AD, Sedlinsky C, Gangoiti MV, Arnol V, Bruzzone L. Metformin reverts deleterious effects of advanced glycation end-products (AGEs) on osteoblastic cells. Exp Clin Endocrinol Diabetes 2008;116:333-40.

[46] Sena CM, Matafome P, Louro T, Nunes E, Fernandes R, Seica RM. Metformin restores endothelial function in aorta of diabetic rats. Br J Pharmacol 2011;163:424-37.

[47] Bonewald LF. The amazing osteocyte. J Bone Miner Res 2011;26:229-38.

[48] Schaffler MB, Kennedy OD. Osteocyte signaling in bone. Curr Osteoporos Rep 2012;10:118-25.
[49] Ma YL, Zeng QQ, Porras LL, Harvey A, Moore TL, Shelbourn TL, et al. Teriparatide [rhPTH (1-34)], but not strontium ranelate, demonstrated bone anabolic efficacy in mature, osteopenic, ovariectomized rats. Endocrinology 2011;152:1767-78.

[50] Jeyabalan J, Viollet B, Smitham P, Ellis SA, Zaman G, Bardin C, et al. The anti-diabetic drug metformin does not affect bone mass in vivo or fracture healing. Osteoporos Int 2013;24:2659-70.

[51] Lipscombe LL, Jamal SA, Booth GL, Hawker GA. The risk of hip fractures in older individuals with diabetes: a populationbased study. Diabetes Care 2007;30:835-41.

[52] Halban PA, Polonsky KS, Bowden DW, Hawkins MA, Ling C, Mather KJ, et al. Beta-cell failure in type 2 diabetes: postulated mechanisms and prospects for prevention and treatment. Diabetes Care 2014;37:1751-8. 